\title{
An Unusual Treatable Cause of Reversible Cardiomyopathy: Vitamin B12 Deficiency
}

\author{
R.Nhiri*, N.ElOuafi \\ Department of Cardiology, University Hospital of Mohammed VI, University Mohammed the First, Oujda, \\ Morocco
}

*Corresponding Author: R.Nhiri, Department of Cardiology, University Hospital of Mohammed VI, University Mohammed the First, Oujda, Morocco

\begin{abstract}
Dilated cardiomyopathy is characterized by a dilated and poorly functioning left ventricle and can result from several different etiologies including ischemic, infectious, metabolic, toxins, autoimmune processes or nutritional deficiencies, cobalamin deficiency-induced cardiomyopathy is an uncommon cause of dilated cardiomyopathy that can go untreated if not considered. To date, there is no case of dilated cardiomyopathy associated with vitamin B12 deficiency was reported in the literature. Here, we describe a 63-year-old male, known hypertensive well balanced under treatment, who was admitted in our hospital for a congestive heart failure with dyspnea (NYHA class IV), orthopnoea and bilateral ankle swelling. He was a 10 pack year smoker. Transthoracic echocardiogram showed dilated cardiomyopathy with a left ventricular ejection fraction (LVEF) of $29 \%$ (biplane simpson's method), with global hypokinesia. There was no significant valvular heart disease. The patient has no evidence suggesting one of the frequent causes of dilated cardiomyopathy. The workup of our patient reveals a low level of vitamin B12. As therapeutic intervention, nutritional supplementation of cobalamin is associated with the classical heart failure therapy, Four month after supplementation, his symptoms have improved with no clinical evidence of heart failure, normalized serum Brain natriuretic peptide (BNP), and normal left ventricular dimension and function on electrocardiography, his ejection fraction has improved from $29 \%$ at presentation to $60 \%$.
\end{abstract}

Keywords: Vitamin B12 deficiency, reversible dilated cardiomyopathy, left ventricular dysfunction.

\section{INTRODUCTION}

Dilated cardiomyopathy can be caused by a variety of disorders. The most frequent causes are coronary artery disease, infectious myocarditis, deposition diseases such as Hemochromatosis and amyloidosis, and medications, in particular chemotherapeutic agents or recreational drugs like alcohol and cocaine. In about $50 \%$ of cases, however, no etiology can be found and the cardiomyopathy is deemed idiopathic. [1]

Cobalamin deficiency causes a wide range of hematological, gastrointestinal, psychiatric and neurological disorders. [2] Dilated cardiomyopathy is an unusual manifestation of cobalamine deficiency.

We report a case of a reversible dilated cardiomyopathy associated with cobalamine deficiency.

\section{CASE REPORT}

A 63-year-old male, known hypertensive well balanced under treatment, ex-smoker weaned 20 years ago, without notion of alcoholism and known family history of cardiomyopathy. Who was admitted in our hospital for dyspnea (NYHA class IV), and orthopnoea. Clinical examination revealed features of congestive cardiac failure, including a peripheral oedema and bilateral lung crepitations. Blood pressure was $138 / 86 \mathrm{mmHg}$. Electrocardiography showed a heart rate at $100 \mathrm{bpm}$, QRS duration was within normal limits. Chest radiograph revealed cardiomegaly and increased pulmonary vascularity. Brain natriuretic peptide (BNP) was raised. Serum troponin and inflammatory markers were normal. Transthoracic echocardiogram showed dilated cardiomyopathy with a left ventricular ejection fraction (LVEF) of 29\% (biplane simpson's method), and global hypokinesia. There was no significant valvular heart disease (figures: 1and 2). He was treated with intravenous furosemide with clinical improvement. Cardiac catheterisation demonstrated angiographically normal coronary arteries. The clinical history and the examination reveal no evidence for a systemic 
immunologic disease; The laboratory analysis show a low level of vitamin B12 $(<83 \mathrm{pg} / \mathrm{ml}$, $\mathrm{NV}: 187-883 \mathrm{pg} / \mathrm{ml})$ without anemia. Vitamin B1, folic acid, vitamin D and ferritin were normal, protein electrophoresis had not objectified a monoclonal bands. Thyroid

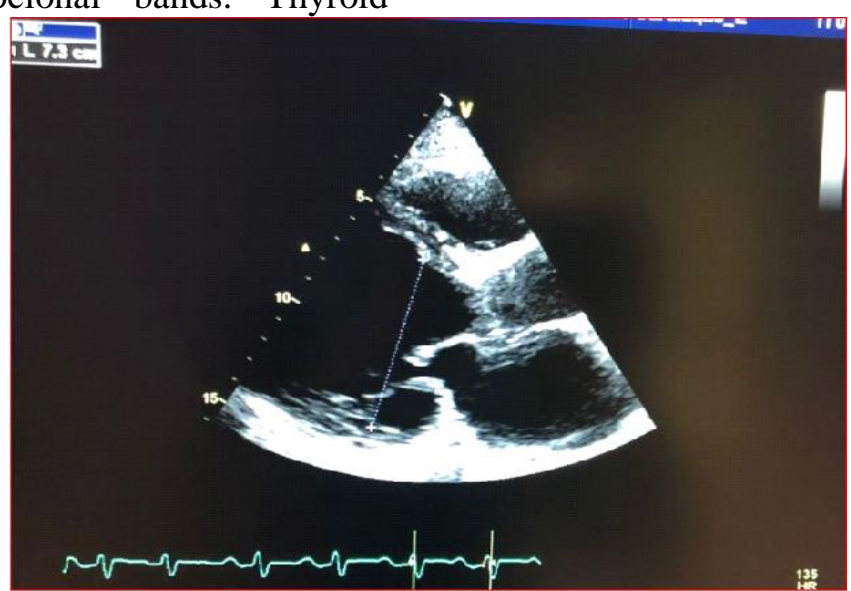

Figure1: Transthoracic Echocardiography in Parasternal Long Axis View Shows Left Ventricle Dilation

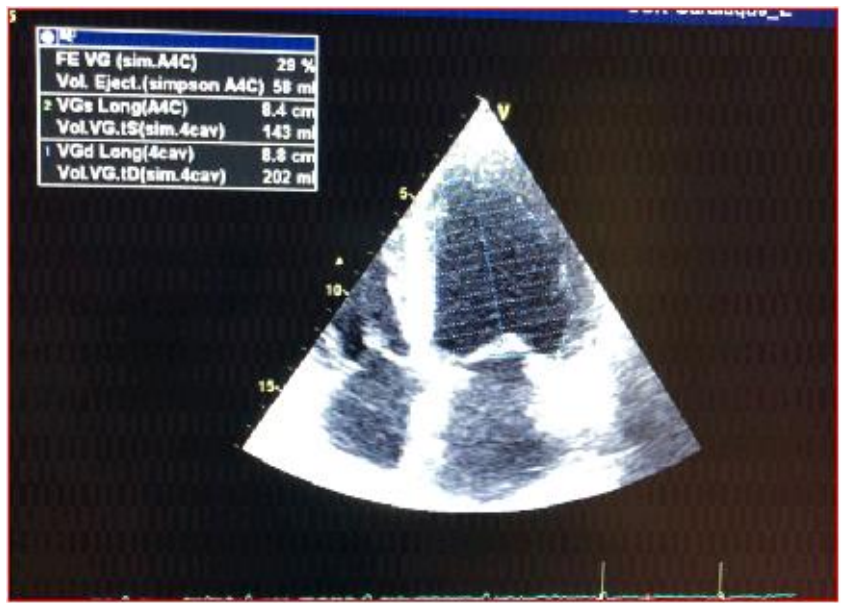

Figure2: Apical 4 Chamber View Shows a Severe Left Ventricle Dysfunction (LVEF At 29\%)

As therapeutic intervention, supplementation of vitamin B12 is associated to the classical heart failure therapy. Four months from initial presentation his symptoms have improved with no clinical evidence of heart failure, normalised hormone values are normal (TSHus: $1.28 \mathrm{mUI} / \mathrm{l}$, $\mathrm{VN}: \quad 0.27-4.2 \mathrm{mUI} / \mathrm{L})$. On serum chemistry profil: Calcium and liver function test are without abnormalities, a part from moderate renal insufficiency. 
The striking change in cardiac function after resolution of cobalamin deficiency in the present case after supplementation of vitamin B12, and exclusion of common causes of reversible cardiomyopathy suggests that the cardiomyopathy was attributable to vitamin B12 deficiency.

As part of the etiological assessment of vitamin B12 deficiency, an assay of anti-parietal cell and intrinsic factor antibodies was requested returning positive, the FOGD was indicated in search of a biermer's disease, but refused by the patient.

\section{DISCUSSION}

The heart requires a continuous supply of energy-providing substrates and amino acids in order to maintain its function (4)Several micronutriments are essential cofactors of metabolic reactions and contribute to the efficiency and the appropriate utilization of energy [9]

Vitamin B12 deficiency causes a wide range of hematological, gastrointestinal, psychiatric and neurological disorders. The various clinical manifestations can be explained on the basis that Vitamin B12 functions as an important cofactor in various biochemical reactions $[(7,2]$

Vitamin B6, B12 and B9 all tend to reduce levels of homocystein, which is associated with increased oxidative stress [8],elevated total homocystein concentrations are mainly caused by folate and vitamin B12 deficiency, and Total homocystein has been linked to the severity of chronic heart failure[5].

Several nutritional deficiencies have been reported in patients with dilated cardiomyopathy, and some of them are involved in the pathogenesis of heart failure [6.4,3,9].

Dilated cardiomyopathy can be caused by a variety of disorders. The most frequent causes are coronary artery disease, infectious myocarditis, deposition diseases such as Hemochromatosis and amyloidosis, and medications, in particular chemotherapeutic agents or recreational drugs like alcohol and cocaine . In about $50 \%$ of cases, however, no etiology can be found and the cardiomyopathy is deemed idiopathic [1].

Our patient has no evidence one of the frequent causes of dilated cardiomyopathy.

An infections cause of dilated cardiomyopathy seems to be unlikely, in the absence of elevated myocardial enzymes and with a negative Creactive protein (CRP) The clinical history and the examination reveal no evidence for a systemic immunologic disease,

echocardiography images and blood chemistry give no clues for a deposition disease like hemochromatosis (ferritin was normal: 74.54 Ug/l, VN: 20-200ug/l) or amyloidosis (protein electrophoresis without monoclonal bands), thyroid hormone values are normal.

The diagnostic workup of our patient reveals a low level of vitamin B12, and a normal value for others Vitamins especially: B1, B6, B9 and D.

Vitamin B12 deficiency biologically manifested by increased methylmalonic acid (MMA) and homocysteine

Homocysteine, methylmalonic acid (MMA), and carnitine are not measured, because not available in our laboratory.

Common causes of reversible dilated cardiomyopathy were effictivelly excluded in this case The improvement of cardiac function after resolution of cobalamin deficiency in the present case suggest that the cardiomyopathy was attributable to vitamin B12 deficiency.

\section{CONCLusion}

The aim of this case report was to show the importance to research treatable cause of dilated cardiomyopathy; vitamine B12 deficiency is an uncommon but potentially reversible cause of dilated crdiomyopathy; To date, there is no case of dilated cardiomyopathy associated with vitamin B12 deficiency was reported in the literature. this present work constitutes the first case of reversible dilated cardiomyopathy due to vitamin B12 deficiency.

\section{REFERENCES}

[1] Adam Ogna. Dilated Cardiomyopathy. Case Report 2. ZKE-Zertifikatkurs Klinische Ernährung . Ospedale La Carità, Locarno

[2] Ch,Briani, Ch,D,Torre,V,Citton,R,Manara,S,Po mpanin,G,Binotto, and F,Adami Cobalamin Deficiency: Clinical Picture and RadiologicalFindings.Nutrients. 2013 Nov; 5(11): 4521-4539.

[3] V,Marinescu, PA McCullough. Expert Rev Cardiovasc Ther. 2011 Sep;9(9):1161-70. doi: 10.1586/erc. 11.95.

[4] Soukoulis V, Dihu JB, Sole $M$ et al. Micronutrient deficiencies an unmet need in heart failure. J Am Coll Cardiol 2009;54(18): 1660-1673. 
[5] M,Herman, et al.Plasma B vitamins and their relation to the severity of chronic heart failure. The American Journal of Clinical Nutrition, Volume 85, Issue 1, 1 January 2007, Pages 117-123, https://doi. org/10.1093/ajcn/85.1.117

[6] Aquilani R, Opasich C, Gualco A et al. Adequate energy-protein intake is not enough to improve nutritional and metabolic status in muscle-depleted patients with chronic heart failure. Eur J Heart Fail 2008;10(11):1127-1135

[7] M,A, Poovathingal, G,George, G,Panicker, S,Abhilash. A clinical Pandora: Unusual manifestations of Vitamin B12 defciency. Department of General Medicine, Amala Institute of Medical Sciences, Thrissur, Kerala, India.. Int J Adv Med Health Res [serial online] 2015 [cited 2018 May 13];2:106-8.

[8] KK, Witte, AL ,Clark, JG Cleland .chronic heart failure and micronutrients. J Am Coll Cardiol. 2001 Jun 1;37(7):1765-74.

[9] E Sciatti, C Lombardi,* A Ravera, et al. Nutritional Deficiency in Patients with Heart Failure. Nutrients. $2016 \mathrm{Jul} ; 8(7)$ : 442.

Citation: R.Nhiri, N.ElOuafi, An Unusual Treatable Cause of Reversible Cardiomyopathy: Vitamin B12 Deficiency. ARC Journal of Cardiology. 2020; 6(2): 14-17. Doi:doi.org/ 10.20431/2455-5991.0602003.

Copyright: (C) 2020 Authors. This is an open-access article distributed under the terms of the Creative Commons Attribution License, which permits unrestricted use, distribution, and reproduction in any medium, provided the original author and source are credited. 